\title{
LAS CONTRADICCIONES DE LA EXPANSIÓN GANADERA EN LAS FRONTERAS NORTE Y SUR DE MÉXICO (ESTADOS DE SONORA Y CHIAPAS)
}

\author{
Por \\ Danicl Villafuerte Solis* \\ Jose Luis Pontigo Sánchez*
}

\begin{abstract}
RESUMEN
En este artículo se plantean las principales contradicciones derivadas del proceso de expansión de la ganadería bovina en México, y de manera particular en los estados de Sonora y Chiapas, los cuales son ejemplos representativos de lo que ocurre en las fronteras norte y sur de nuestro país en relación al fenómeno que se ha dado en llamar ganaderización o ganaderización de la agricultura.
\end{abstract}

\begin{abstract}
This article establishes the most important contradictions from the catte raising in Mexico and particularly in the states of Sonora and Chiapas, which are examples of what is happenning in Mexican North and South borders in relation to the phenomenon called cattling or "agriculture cattling".
\end{abstract}

El crecimiento y crisis, como dos polos de la dinámica que caracterizan hoy día a la ganadería mexicana, han dado como resultado que numerosos académicos e instituciones con diversos enfoques se interesen por el estudio de la ganadería en México ${ }^{1}$. Estos estudios encuentran justificación no sólo por la importancia que ha adquirido la ganadería en la economía regional, sino además, y en principio, por la dinámica que presenta, que comprende desde los elementos estrictamente productivos, con su entorno ecológico, hasta las cuestiones socioeconómicas y políticas. Es en esta perspectiva donde este trabajo pretende aportar elementos de comparación sobre las características particulares del fenomeno que se ha dado en llamar "ganaderización" o "ganaderización de la agricultura" en dos espacios que difieren substancialmente en términos de sus recursos

\footnotetext{
- Investigadores del Centro de Investigaciones Ecológicas del Sureste (CIES).

1 Dentro de los estudios más recientes podemos citar los siguientes: El proceso de instifucionalización de la ganadería bovina de carne en México (Cnu, 1978); Ganadería y estructura agraria en Chiapas (Femández y Tarrío, 1983); El desarrollo agroindustrial y la ganadería en México (Feder y otros, 1982); Agricultura y ganadería. Competencia por el uso de la tierra (Pérez Espejo, 1987).
} 
naturales, situación geopolítica, tradición cultural y estructura económica, esto es, los estados de Sonora y Chiapas, los cuales comparten estrechas relaciones de intercambio comercial y cultural con los Estados Unidos y Guatemala, respectivamente.

Como se sabe, desde mediados de los afios cincuenta, la ganadería y la industria de la carne experimentan profundas transformaciones a nivel internacional, destacando Estados Unidos, que se convierte en el primer productor y exportador de carnes rojas en el mundo. Este país ha logrado, mediante innovaciones tecnológicas, acortar el ciclo de producción pecuaria, obteniendo el producto en menor tiempo para lanzarlo al mercado. También desde mediados de los años sesenta los países industrializados, en especial Estados Unidos, aportan considerables sumas de capital y tecnología a los países del tercer mundo para impulsar la actividad ganadera, a fin de satisfacer la demanda internacional de alimentos y, por otro, controlar la producción y distribución de productos pecuarios ${ }^{2}$. A partir de este periodo de "revolución ganadera", en México se da una expansión de la ganadería bovina, resultado no sólo del apoyo externo, sino del proceso mismo del mercado interno: industrialización, crecimiento demográfico y de ingreso de ciertas capas de la sociedad, etc. Dicho en otros términos, la expansión es inducida tanto por la dinámica concreta de la reproducción del capital nacional, como por las exigencias del mercado mundial de la carne que reasigna a estas regiones nuevos papeles en la división internacional del trabajo, como el de ser proveedoras de proteínas baratas para una parte de la población de los países centrales (Fernández y Tarrío, 1983:10).

Según fuentes estadísticas, se estima que en 1960 existía en el país una población bovina de 17.6 millones de cabezas y para 1980 se incrementa a 34.5 millones, siendo fundamentalmente la región tropical donde se

2 En la década de los sesenta, y tras la falacia de la "vocación ganadera del trópico", cobra impulso la promoción de la producción ganadera, no sólo en el trópico sino en diversas regiones de América Latina. Así, en Bolivia, el Banco Interamericano de Desarrollo (BD) autorizó un préstamo de 5 millones de dólares más un desembolso del gobiemo boliviano por 7 millones de dólares. En Brasil, el BIRF autoriz6 40 millones de dólares, más un desembolso del gobiemo brasileño por la misma cantidad, con el objeto de desarrollar zonas ganaderas. Por su parte, el BD autorizo en 1969 un préstamo de 26 millones de dólares más un aporte local por igual cantidad, el objetivo era el financiamiento de un programa de crédito destinado a incrementar la producción de came vacuna en los estados de Bahías, Minas Gerais y Espíritu Santo. En Colombia, con asistencia del BID, del BIRF y de ayuda unilateral, se fomenta la producción de came vacuna en la costa atlántica. En Ecuador, con un préstamo del BIRF, se pretenden utilizar 480,000 hectáreas para la producción de carne vacuna y lácteos. Asimismo, desde principios de 1960, los países centroamericanos inician programas de desarrollo ganadero para la exportación, principalmente al mercado norteamericano, con préstamos de la AIF. (Véase García, 1983). 
reportan los más altos índices de crecimiento y expansión ganadera, región que se va especializando en la producción de carne vacuna para el abastecimiento del mercado nacional.

Por otro lado, se estima que la superficie dedicada a la ganadería es de 82 millones de hectáreas, aunque si se agregan 46 millones de hectáreas clasificadas como agostaderos de menores rendimientos, suman un total de 128 millones de hectáreas ocupadas por la ganadería, de las cuales un $24.2 \%$ de la superficie se destinó en 1985 a cultivos vinculados con la alimentación del ganado menor y mayor, tales como sorgo, maíz forrajero, oleaginosas y praderas cultivadas (Chauvet, 1987:49).

Ahora bien, si se analiza el crecimiento de la ganadería por regiones, se observa que éste ha sido más dinámico en el sureste del país, particularmente en la parte del trópico húmedo, donde se han afectado vastas extensiones de bosques y selvas para la expansión de pastizales. Tan sólo de 1970 a 1979 los pastos naturales del trópico se triplicaron, al pasar de 5 millones 17 mil hectáreas a 15 millones 841 hectáreas; la región norte es, hasta la fecha, la que ocupa las superficies de agostadero más grandes del país: alrededor de 90 millones de hectáreas que representan el $75 \%$ del total de pastos nacionales. En esta región hubo un incremento global de los pastos naturales del $50 \%$ en la última década, pues para 1970 contaba con casi 60 millones de hectáreas; la región centro del país cuenta con una superficie de pastos similar a la del trópico: 15 millones 23 mil hectáreas para 1979, es decir, en este caso, desde el inicio de los setenta la superficie de pastos se incrementó en aproximadamente 50\% (Camou y Pérez, 1985:39-40).

Sin embargo, el fenómeno de la ganaderización en México no se explica únicamente por el incremento de las áreas de pastizales en el país y en sus distintas regiones, sino también porque ha modificado el uso del recurso tierra en favor de cultivos forrajeros. En efecto, desde los años sesenta se observan cambios acelerados de la superficie cosechada con cultivos cuyo fin es la alimentación del ganado, en detrimento de la superficie con cultivos básicos para la población humana.

La expansión ganadera $\chi$ el crecimiento de los cultivos forrajeros no sólo ha desplazado la producción de granos básicos, sino también se ha extendido sobre las áreas forestales y las superficies que antes estaban ocupadas por cultivos tradicionales de exportación como el algodón, la caña de azúcar, el cacao y el café, que además en los últimos años han sufrido bajas en el mercado internacional.

En este sentido, puede afirmarse que una parte de la agricultura se subordina al desarrollo de la ganadería, que a su vez está fuertemente vinculada a la industria. Es decir, el dinamismo de los cultivos de consumo 
animal revela que el sector agrícola responde cada vez más a las necesidades de la industria productora de alimentos balanceados para el ganado, misma que esta fuertemente controlada por las empresas transnacionales como Ralston Purina, Anderson Clayton, International Multifoods, etc., que, además de controlar otros insumos industriales, son propietarias de granjas avicolas intensivas.

Durante más de dos décadas la ganadería registró un crecimiento inusitado, tanto por la ampliación de la superficie ocupada como por el número de cabezas. Sin embargo, a partir de los affos ochenta, este subsector entra en crisis como resultado del agotamiento del modelo extensivo de producción, que tiene que ver con las limitaciones del recurso tierra, con los conflictos agrarios y con la estructura de precios.

En este sentido, la participación de la ganaderfa en el producto interno bruto (PIB) no sólo es menor que el de la agricultura, sino que cada vez disminuye más, pasando del 40.7 por ciento en 1960 al 34.4 por ciento en 1985. En efecto, la crisis económica, que se agudizó a partir de 1982, ha impactado severamente la producción ganadera, presentando en los últimos affos ${ }^{3}$ un debilitamiento del mercado interno debido a los precios y al deterioro del ingreso de ciertas capas de la población y a la contracción del empleo que provoca una tendencia a la baja en la demanda de carne.

Según fuentes oficiales (Estadísticas de la SARH y Cuarto Informe de Gobierno del Lic. Miguel de la Madrid), la ganadería bovina tiene un crecimiento sostenido hasta 1983, llegando a $37.5 \%$ millones de cabezas; a partir de ese affo, el inventario ha venido disminuyendo, en 1984 se registraron 30.4 millones de cabezas y en 198531.4 millones.

La crisis ganadera se ve agudizada aún más por los problemas de sequía e incendios que se propician año con affo en todo el país, incidiendo fuertemente en el deterioro y escasez de forrajes. En 1988 la zona más afectada por la sequía fue la región norte, donde ya de por sí la escasez de lluvias y el agotamiento del suelo, ocasionado por el sobrepastoreo, han impactado fuertemente en los grados de erosión.

Es en esta serie de procesos contradictorios donde tiene lugar el crecimiento de la ganadería en las fronteras norte y sur de México que mucho tienen en común, pero, al mismo tiempo, difieren por el papel que cumplen dentro de la división territorial del trabajo.

3 Durante 1988, segín reporte del Banco de México, la actividad ganadera disminuy6 $2.7 \%$, destacando en particular, las contradicciones en la producción de leche y en la oferta interna de came, Esta por segundo año consecutivo. Más del $50 \%$ de la came consumida en el Distrito Federal durante el año pasado fue de importación, y si en el país no se presentaron problemas de escasez fue debido a la liberación del comercio exterior de estos productos. (Uno más Uno, 3 de mayo de 1989:15). 


\section{ALGUNAS CARACTERf́sticas de LA PRODUCCIÓN DE BOVINOS EN LA ZONA NORTE}

El crecimiento que ha experimentado la ganadería en el norte de México durante los últimos treinta y cinco años se ha fincado en la ocupación de grandes extensiones de tierra. Esta característica, propia de la ganadería extensiva, ha provocado un agudo proceso de saturación de los pastizales, traduciéndose en un deterioro paulatino de los mismos. Esta situación ha sido evidenciada por algunas investigaciones que se han realizado en torno a la ganadería norteffa (CBPAL, 1973; COPARMEX, 1965).

La tendencia al sobrepastoreo se agudiza en la medida en que las ganancias generadas en las empresas no se traducen en inversiones que permitan elevar la capacidad productiva de los pastos, situación que se ve agudizada por la escasa incorporación de prácticas de manejo que conlleven a evitar, en mayor medida, el deterioro de los pastizales.

Las bajas inversiones de capital fijo hacen que el medio fundamental en la producción de ganado sea la tierra. En la región se estima que los indices de agostadero varian de 15 a 50 hectáreas por cabeza de ganado, de tal manera que desde el punto de vista de la legislación agraria, la pequefia propiedad podría tener entre 7,500 y 25,000 hectáreas.

Es indudable que los patrones de producción en el norte se encuentran estrechamente relacionados con los factores de orden físico, dando origen a una relación pasto-agua-tierra bastante desfavorable para la producción ganadera. Sin embargo, es importante seffalar que la irracionalidad del proceso productivo ganadero, que se evidencia en las bajas inversiones de capital en las explotaciones, no permite una transformación sustancial en las condiciones del medio fisico, por lo que los actuales patrones de producción no pueden explicarse exclusivamente a partir de las condiciones del medio natural, sino que además hay que tomar en cuenta los factores de orden económico, social y político.

Históricamente el norte de México ha tenido un papel definitivo dentro de la región. Ya desde la segunda mitad del siglo XIX Estados Unidos se había convertido en el principal mercado de productos agropecuarios mexicanos; sin embargo, aunque las exportaciones de ganado vivo eran considerables, el comercio de pieles constituía el componente principal del valor de las exportaciones de estos productos. En este sentido, Lopez Rosado (1977) seffala que en el periodo 1885-1900 las exportaciones de ganado en pie registradas fueron 1'170,000 animales, o sea cerca de 234,000 anuales.

En la última parte del mismo siglo la importancia de la ganadería había crecido considerablemente; a nivel comercial se estaba en presencia de una 
coyuntura favorable que permitia a los ganaderos obtener jugosas ganancias, pues varios países de Europa, incluso los Estados Unidos, tradicionales consumidores de carne, presentaban crecientes déficits en su producción, lo que obligaba a realizar importaciones. De esta manera, el comercio se hacía más importante, particularmente en las últimas décadas, sin embargo, las medidas arancelarias impuestas por el gobierno norteamericano impedían un crecimiento armónico de la ganadería norteffa.

No obstante, durante la última parte del siglo pasado y principios de éste, el comercio exterior de la ganadería mexicana se vio favorecido tanto por las políticas del Estado como por los precios del mercado intemacional. En estas condiciones, el mercado externo coadyuvó a profundizar las diferencias en la producción, entre las ganaderías del centro y sur con la de los estados fronterizos del norte; así, mientras las primeras se caracterizaban por poseer un sistema tradicional, las segundas eran más dinámicas, incluso a nivel genético presentaban diferencias notables.

En términos generales, se puede decir que la producción ganadera en los estados nortefios va presentando un proceso de acondicionamiento para el comercio con el vecino país. De esta manera, el ganado se convierte en una mercancía con características específicas: raza, edad y peso. Para los ganaderos norteamericanos, el desarrollo de la ganadería del norte de México significaba una fuente de fácil acceso para el abastecimiento de ganado para la engorda, y para los ganaderos nortefios Estados Unidos representaba un mercado con mayores ventajas respecto al mercado interior, fundamentalmente por la diferencia de precios.

Hasta 1946 se observa un importante flujo comercial de ganado con los Estados Unidos. A pesar de las variaciones en las tarifas arancelarias, que provocaron ciertas oscilaciones en el comercio, se puede decir que en general las exportaciones presentaron un comportamiento más o menos estable. Sin embargo, el periodo de bonanza comercial pronto llego a su fin, los brotes de la fiebre aftosa aparecen a fines de 1946 y con ello la frontera comercial de ganado con los Estados Unidos se cierra.

En 1954 se declara oficialmente que México ha quedado libre de la aftosa y, en consecuencia, se reanudan las exportaciones de ganado en pie. La gran dinamicidad que adquiere la exportación de ganado en pie después de la aftosa coincide plenamente con el desarrollo de la engorda de ganado en gran escala en el sureste de los Estados Unidos. En esta perspectiva, el norte de México se convierte en el principal abastecedor de becerros para la engorda en el vecino país.

Esta relación de dependencia comencial de México con los Estados Unidos ha sido uno de los factores que han contribuido de manera determinante a la especialización de la zona norte de México en la producción 
de becerros para la engorda. Además, la demanda selectiva de ganado ha conducido a que los ganaderos se preocupen más por el mejoramiento genético que por los aspectos de manejo de la empresa, particularmente en lo que se refiere a pastizales.

\section{EL PROCESO DE GANADERIZACIÓN EN SONORA}

El acelerado crecimiento de la ganadería sonorense forma parte de un amplio proceso que se viene dando a nivel nacional a partir de los affos cincuenta. Este rápido crecimiento de la ganadería, que se ha dado en llamar proceso de ganaderización, asume rasgos particulares en el estado de Sonora por las características de sus recursos naturales, principalmente del complejo agua-suelo-pasto, por su conformación histórica y su relación de vecindad con los Estados Unidos. Si entendemos el proceso de ganaderización como la expansión de la frontera ganadera, tenemos por ejemplo que en las regiones tropicales del sur del país ha significado la desforestación de vastas extensiones de selva, provocando la degradación del medio ecológico. En el caso de Sonora, dada la particularidad de su clima árido, el proceso ha implicado la ocupación de enormes superficies sin necesidad de realizar desmontes, pues en muchos casos la poca vegetación existente sirve de alimento al ganado.

La ganaderización en Sonora también ha significado cambios importantes en el uso del suelo, incluso en el sector ejidal, en donde con anterioridad se dedicaban a las actividades agrícolas de temporal hoy producen becerros. A nivel socioeconómico, las implicaciones más significativas de este proceso son: el reforzamiento de la dependencia con los

\section{CUADRO 1. Evolución de la población ganadera y la superficie de pastos en Sonora.}

\begin{tabular}{ccc}
\hline Affo & No. de cabezas & Sup. de pasto (has.) \\
\hline & 611157 & 4735752 \\
1940 & 885381 & 7188945 \\
1950 & 1340803 & 9812831 \\
1960 & 1450791 & 10196001 \\
1970 & 1600000 & 15600000 \\
1982 & 1585000 & 17000000 \\
& \\
\hline & \\
\hline
\end{tabular}


Estados Unidos, la polarización de la estructura agraria y el desplazamiento de la población humana de las zonas ganaderas a los centros de mayor actividad económica.

Por su extensión territorial, Sonora ocupa el segundo lugar en el país después de Chihuahua. La enorme extensión, que suma casi 18.5 millones de hectáreas, junto con las particulares condiciones climáticas, en donde no existe la posiblidad de ampliar la frontera agrícola sino a condición de fuertes inversiones en infraestructura de riego, han propiciado que la ganadería se expanda rápidamente.

La ganaderización en Sonora no sólo se evidencia en el crecimiento de la población bovina y la ampliación de la superficie de agostadero; hay que seffalar, además, que en el ámbito del sector agrícola se producen cambios importantes en el uso del recurso tierra: por un lado, la ocupación directa de algunas áreas de labor por pastos cultivados, significando en números absolutos poco más de 30 mil hectáreas, de las cuales 3,393 se ubicaban en áreas de riego; por otro lado, se incrementan las áreas destinadas al cultivo de productos forrajeros que son consumidos directamente por el ganado, en este sentido, según cifras censales de 1970, sólo en lo que se refiere a sorgo y alfalfa sumaban 49,597 hectáreas. Finalmente, hay que seffalar la aparición de otros cultivos que se encuentran directamente relacionados con la ganadería, es el caso de las oleaginosas, fundamentalmente cártamo, soya y ajonjolí, cuyos subproductos constituyen materias primas para la elaboración de alimentos balanceados para animales. Para tener una idea de la importancia de estos cultivos basta sef̂alar que de 60,279 hectáreas cosechadas en el ciclo agrícola 1960/1961 se pasa a 325,932 hectáreas en el ciclo 1978/1979, significando un incremento de $440.7 \%$, esto es, más del $40 \%$ de la superficie cosechada en Sonora en ese afio.

Los cambios en el uso del suelo han conducido, en algunos casos, a un proceso de reducción de las áreas dedicadas a cultivos orientados a la alimentación humana, y en otros, a desalentar la producción de éstos. En el primero, resulta ilustrativo el caso del cultivo del arroz, que de ocupar 31,851 hectáreas en 1960, prácticamente desaparece en 1970. En el segundo, se puede observar que los cultivos de maíz y frijol no han experimentado cambios substanciales en cuanto a las superficies ocupadas; así, por ejemplo, en 1950 estos cultivos ocupaban 75 mil hectáreas, sin embargo en 1970 sólo llegan a poco más de 41 mil.

Una de las contradicciones más evidentes del proceso de ganaderización es que, no obstante la enorme superficie que ocupa la ganadería bovina, ésta va experimentando un rápido descenso dentro del valor de la producción del subsector pecuario; así, de 45\% que representaba en 1978 
pasa a significar solamente el $34.4 \%$ en 1980 . Esta pérdida de dinamicidad de la ganadería bovina no es un fenómeno exclusivo de Sonora, se trata, como se ha seffalado, de un problema nacional en donde se puede apreciar que el patrón de crecimiento ha entrado en crisis, la cual se refleja no sólo en la producción sino también en la productividad.

Finalmente, hay que decir que el proceso de ganaderización en Sonora ha implicado también el reforzamiento de la tendencia al rentismo de parcelas, principalmente en determinados procesos productivos como por ejemplo la llamada preengorda. Este fenómeno trae como consecuencia una fuerte presión sobre los agostaderos, causando serios deterioros. En este sentido hay sefialamientos que indican que, de seguir la tendencia actual en el uso de los recursos agua-tierra-pasto, llegará el momento en que la actividad ganadera desaparecerá. A nivel oficial, esta problemática se reconoce claramente cuando se señala que: "Debido a la sobrecarga animal de los agostaderos en la entidad, éstos se han deteriorado en proporciones importantes. El sobrepastoreo actual de los agostaderos es de 306\%. De los 15 millones de hectáreas de pastos naturales se estima que una tercera parte de los mismos se encuentran en diferentes grados de erosión, en algunos casos ya muy avanzados" (Gobierno del Estado de Sonora. Plan de Desarrollo Económico y Social 1980-1985, 1980:17).

\section{NUEVAS MODALIDADES DEL DESARROLLO GANADERO EN SONORA: ¿EXPANSIÓN O RECONVERSIÓN?}

Las fuertes contradicciones que han caracterizado al desarrollo ganadero, las particulares condiciones del medio natural y la fuerte dependencia con el mercado norteamericano han condicionado, desde los inicios de los años setenta, el surgimiento de una ganadería más tecnificada que reclama insumos industriales, esto es, la industria engordadora.

La engorda de ganado es considerada como uno de los procesos más dinámicos de la producción: en él la composición de insumos utilizados tienen un alto contenido industrial, acortando el tiempo de producción. La alimentación del ganado se hace con raciones de alto contenido en granos, pastas oleaginosas y esquilmos agrícolas. La industria engordadora es favorecida por la política oficial, no sólo en lo que respecta al establecimiento de precios diferenciales de la carne, sino también por los subsidios destinados a la alimentación del ganado. Esta política deliberada del gobiemo favorece abiertamente a los grandes engordadores, quienes además poseen o son accionistas mayoritarios de las plantas Tipo Inspección Federal (TIF), en donde se transforma el ganado en canales y cortes especiales, tal es el caso del grupo Mezoro, antes Mesquital del Oro, el 
grupo Valmo y los hermanos Ronquillo, quienes ejercen un fuerte control del proceso de transformación del ganado permitiéndoles elevar el nivel de ganancias. Las empresas de estos grupos presentan un alto grado de integración, en donde el ganado es aprovechado en su totalidad obteniéndose subproductos como vísceras, sebos y pieles que son comercializados bajo otros canales.

Información oficial revela que en 1980 existían 57 empresas engordadoras en el estado con una capacidad instalada de 149,350 cabezas, concentrándose en el municipio de Cajeme el $43.8 \%$ de los establecimientos, aunque la capacidad instalada sólo representó el 16\%; en cambio, el municipio de Hermosillo, con el $28.1 \%$ de los establecimientos, representó el $60.2 \%$ de la capacidad instalada (Carrillo, 1988:153). Para 1981, la capacidad instalada de las engordas en el estado presenta un importante incremento al pasar a 185,750 cabezas, lo que significó en términos relativos $24.3 \%$ respecto a 1980 . Este crecimiento estuvo sustentado por el aumento en el número de engordas en los municipios de Hermosillo y Cajeme, pasando el primero de una capacidad de 89,950 cabezas a 118,850 , y el segundo de 23,900 a 28,900 cabezas; con este crecimiento Hermosillo concentró el $64 \%$ de la capacidad de engordar en el estado y Cajeme el $16 \%$.

Es importante hacer notar que la producción de ganado en corral acusa un alto grado de concentración. En efecto, para 1980 el $41 \%$ de la CUADRO 2. Evolución de la capacidad instalada de los corrales de engorda en Sonora.

\begin{tabular}{lrr}
\hline Municipio & 1980 & 1981 \\
\hline & 89950 & 118850 \\
Hermosillo & 1500 & 1500 \\
Nogales & 2500 & 2500 \\
S. L. Río Colorado & 5000 & 5000 \\
Bácum & 23900 & 28900 \\
Cajeme & 8000 & 8000 \\
Guaymas & 7500 & 8000 \\
Navojoa & 1000 & 1000 \\
Huatabampo & 6000 & 6000 \\
Carbó & 3000 & 3000 \\
Rosario & 3000 & 3000 \\
Caborca & 149350 & 185750 \\
Total & & \\
\hline
\end{tabular}

FUENTE: Unión Sonorense de Engordadores de Ganado, A.C.; Plan de Desarrollo Agropecuario y Forestal del Estado de Sonora 1982- 1988. 
capacidad instalada es detentada por sólo tres engordadores de los 57 registrados; asimismo poseían el $42.7 \%$ del valor total de las instalaciones. Esta situación no sólo plantea el problema de la concentración de los recursos financieros e insumos, sino también de la influencia que ejercen en el mercado en la regulación de la oferta y los precios.

De acuerdo al tiempo de engorda del ganado, los corrales pueden ser utilizados hasta tres veces al año, sin embargo, en Sonora normalmente se realizan dos ciclos y obedece fundamentalmente a que no existe una oferta adecuada de ganado. Si consideramos que la capacidad instalada de los corrales en el estado ascienden a 185,750 cabezas en 1981, en dos ciclos significaría una demanda de 371,500 cabezas de ganado. Responder a la demanda de la industria engordadora implicaría suprimir las exportaciones de ganado en pie, lo que agravaría aún más la ya difícil situación que pressentan los agostaderos, pues como se sabe el ganado que entra en los corrales rebasa los $200 \mathrm{kilos}$, implicando retener el ganado más tiempo en los agostaderos.

\section{CUADRO 3. Distribución del valor de los corrales de engorda en Sonora 1980.}

Nombre de la empresa $\quad$ Miles de pesos $\%$

$\begin{array}{lrr}\text { Engorda, S.A. de C.V.* } & 120000 & 20.9 \\ \text { Ganadera Valmo, S.A. } & 75000 & 13.1 \\ \text { Sergio Torres } & 50000 & 8.7 \\ \text { Total } & 245000 & 42.7 \\ \text { Resto del estado } & 329250 & 57.3 \\ \text { Total Sonora } & 574250 & 100.0\end{array}$

FUENTE: Subsecretaría de Ganadería.

*= Propiedad del grupo Mezoro.

Pero el problema de la industria engordadora no sólo se debe a la falta de ganado, sino también a otros factores como la escasez de recursos financieros y la demanda de insumos para la alimentación del ganado, particularmente en lo que se refiere a granos. De acuerdo a la información disponible, en 1980 la engorda de ganado implicó un consumo de maíz de 168,000 toneladas, esto representa el $17.9 \%$ del consumo total de maíz por el subsector pecuario del estado, que en números absolutos ascendió a 937,000 toneladas, correspondiendo 450,000 a la avicultura y 270,000 a la porcicultura. El consumo de granos por la ganadería se torna cada vez más crítico en cuanto que éstos son importados de los Estados Unidos o 
CUADRO 4. Principales insumos utilizados en el subsector pecuario del estado de Sonora (Toneladas 1980).

\begin{tabular}{lrrrrrr} 
Producto & U.G.R.S. & $\begin{array}{c}\text { Porci- } \\
\text { cultura }\end{array}$ & $\begin{array}{c}\text { Corrales lecheros } \\
\text { de engorda }\end{array}$ & $\begin{array}{c}\text { Avicul- } \\
\text { tores }\end{array}$ & Total \\
\hline Máz grano & 25000 & 270000 & 168000 & 24000 & 450000 & 937000 \\
Alfalfa & 3000 & 1000 & 30000 & 50000 & - & 84000 \\
Melaza & 2000 & - & 33000 & - & 35000 & 35000 \\
Cártamo & 3500 & 18000 & 50000 & - & 36000 & 107000 \\
Marinolina & 2000 & 7000 & 10000 & - & 3000 & 22000 \\
Soya & 500 & 43000 & - & - & 10000 & 143500 \\
Caj.de algodón & 1800 & - & 18000 & - & - & 19800
\end{tabular}

FUENTE: Departamento Contraloría; Unión Ganadera Regional de Sonora.

de Canadá, como está ocurriendo en el caso de la cebada, pues Sonora no produce lo necesario para cubrir las necesidades de esta industria. Al respecto, hay que observar que a lo largo de los últimos veinte años la producción de maíz en el estado no ha rebasado las 150 mil toneladas, con excepción del año de 1965 en que se obtuvo poco más de 202 mil toneladas.

El consumo de granos por la industria engordadora está directamente relacionado con su crecimiento y con la disponibilidad de estos insumos, sin embargo, se puede observar que, de acuerdo a los precios de la carne, puede

CUADRO 5. Concentración de la capacidad instalada de los corrales de engorda en Sonora 1980.

\begin{tabular}{ccc}
\hline Propiedad & $\begin{array}{c}\text { Capacidad } \\
\text { (cabeza) }\end{array}$ & $\%$ \\
\hline
\end{tabular}

$\begin{array}{lrr}\text { Agromosa* } & 30000 & 20.1 \\ \text { Ganadera Valmo } & 18000 & 12.1 \\ \text { Sergio Torres } & 13000 & 8.8 \\ \text { Total } & 61000 & 41.0 \\ \text { Resto del estado } & 88350 & 59.0 \\ \text { Total Sonora } & 149350 & 100.0\end{array}$

FUENTE: Unión Sonorense de Engordaderos de Ganado, A.C.; Residencia de Planeación.

* = Corresponde a la empresa Engorda, S.A. de C.V., citada en el cuadro 3. 
aumentar o disminuir su consumo; asi, cuando hay buenos precios en el mercado, el productor utiliza un método de engorda rápida, esto implica la utilización de raciones de alto contenido en proteínas (granos, melaza, urea, etc.), con lo cual se estima un aumento de peso de 1.5 kilos por día.

$\mathrm{Si}$ en sus inicios la industria engordadora fue el instrumento para ampliar la demanda de crías, constituyendo un mercado alternativo a la exportación de ganado en pie a los Estados Unidos, hoy se cuestiona, incluso oficialmente, que la producción de carne de esta industria resulta de una calidad innecesaria para satisfacer los requerimientos nutricionales de la población. El consumo de granos por la ganadería debe ser considerado como un problema que afecta a la población de bajos ingresos, pues la transformación de granos a carne no resuelve los graves problemas de desnutrición de grandes sectores de la población ya que la came no forma parte de la dieta popular.

El desarrollo de la industria engordadora también ha implicado un proceso de cambio en los sistemas de transformación y comercialización del ganado. En este sentido, se puede observar que de 1970 a 1983 las cuotas de exportación de ganado en pie han registrado un progresivo decremento.

CUADRO 6. Exportación de ganado bovino a los Estados Unidos. Estado de Sonora.

\begin{tabular}{lc}
\hline Affo ganadero & Cabezas exportadas \\
\hline $1960-1970$ & 254468 \\
$1970-1971$ & 223439 \\
$1971-1972$ & 246520 \\
$1972-1973$ & 200710 \\
$1973-1974$ & 127038 \\
$1974-1975$ & 126627 \\
$1975-1976$ & 74105 \\
$1976-1977$ & 141195 \\
$1977-1978$ & 173928 \\
$1978-1979$ & 134889 \\
$1979-1980$ & 133344 \\
$1980-1981$ & 95809 \\
$1981-1982$ & 83350 \\
$1982-1983$ & 146904 \\
\hline
\end{tabular}

FUENTE: Dirección de Ganadería. Gobierno del Estado. 
CUADRO 7. Sacrificio de ganado bovino en rastros municipales y plantas TIF.

\begin{tabular}{cccccc}
\hline & \multicolumn{2}{c}{ Rastros } & \multicolumn{2}{c}{ Plantas } \\
Afio & Municipales & $\%$ & TIF & $\%$ & Total \\
\hline 1970 & 119379 & 87.07 & 17721 & 12.93 & 137100 \\
1971 & 126161 & 86.97 & 18895 & 13.03 & 145056 \\
1972 & 122552 & 82.82 & 25406 & 17.18 & 147958 \\
1973 & 126670 & 80.51 & 30645 & 19.49 & 157315 \\
1974 & 134550 & 74.64 & 45702 & 25.36 & 180252 \\
1975 & 135570 & 69.09 & 60637 & 30.91 & 196207 \\
1976 & 171410 & 82.51 & 36316 & 17.49 & 207726 \\
1977 & 187331 & 85.67 & 31313 & 14.33 & 218644 \\
1978 & 168775 & 80.23 & 41584 & 19.77 & 210646 \\
1979 & 136137 & 62.55 & 81507 & 37.45 & 217646 \\
1980 & 130243 & 64.77 & 70837 & 35.10 & 201080 \\
\hline
\end{tabular}

FUENTE: Subsecretaría de Ganadería. Gobierno del Estado de Sonora.

La reducción de las exportaciones de ganado en pie presenta una correlación significativa con el incremento de ganado sacrificado en las plantas TIF, donde se transforma en canales y cortes especiales bajo un sistema altamente tecnificado.

\section{LA FRONTERA SUR}

A nivel estatal, la región de la frontera sur de México está formada por los estados de Campeche, Chiapas, Tabasco y Quintana Roo; a nivel municipal está formada por 21 municipios colindantes ya sea con Belice o con Guatemala, de los cuales 16 pertenecen al estado de Chiapas, dos a Campeche, dos a Tabasco y uno a Quintana Roo. Con una superficie aproximada de $84,603.6 \mathrm{~km}$. , la región definida a nivel municipal tuvo en 1988 una población estimada de alrededor de 1'182,800 habitantes, de los cuales cerca del $56 \%$ vivían dentro de los límites de Chiapas; $21 \%$ en Campeche; 15\% en Quintana Roo y 8\% en Tabasco (Carrillo, 1988:153).

La orientación económica principal de la región ha sido hacia las actividades primarias. Por ejemplo, en 1980 alrededor del $60 \%$ de la población económicamente activa (PEA) de la región se dedicaba a actividades primarias, mientras que a nivel estatal, la proporción correspondiente fue de $50 \%$ en 
promedio (Carrillo, 1988:153). "Lo anterior muestra (...) que Chiapas es sin duda el estado más fronterizo de la región. No solamente cuenta con alrededor del 58\% de la frontera mexicana con Centroamérica, sino que sus municipios fronterizos albergan la mayor proporción de la población de la región y se dedicaban a las actividades primarias, también en una mayor proporción"4

Durante los últimos affos, el sureste mexicano, y en particular el estado de Chiapas, ha sido foco de atención de numerosos organismos tanto de carácter oficial como privado. Chiapas, al igual que otros estados que forman la frontera sur, se ha convertido en una región estratégica en cuanto que posee grandes recursos, particularmente en materia de energéticos.

Las implicaciones sociopolíticas y ecológicas derivadas de la irracionalidad en el uso de los recursos a que ha sido sometida la región, por un lado, y por otro, la de plantear alternativas diferentes a este proceso, están detrás del interés manifiesto de las instituciones en desarrollar proyectos de investigación y desarrollo.

A pesar de todo ello, las condiciones que presenta la región, y en particular el estado de Chiapas, siguen siendo esencialmente las mismas. Lo que es más, en los últimos años, con la explotación petrolera y el desarrollo de proyectos hidroeléctricos, la situación de la mayoría de la población se ha tornado difícil. La inflación y el deterioro de las condiciones de vida de grandes sectores son algunos rasgos que caracterizan el momento actual en Chiapas.

\section{EXPANSIÓN GANADERA EN CHIAPAS}

Históricamente la producción ganadera ha jugado un papel significativo en la formación económico-social de Chiapas. Desde el periodo colonial esta actividad ha tenido importancia en varias regiones del estado, superando incluso los rendimientos de los cultivos agrícolas en esos años, como son añil, caña de azúcar y cacao, ejerciendo además una fuerte acción despojadora sobre las tierras de las comunidades indígenas, a quienes se les prohibió la posibilidad de criar ganado mayor a escala comercial. Sin embargo, es a partir de 1940 cuando se observa un poderoso impulso al desarrollo ganadero, llegando en los años recientes a ocupar un lugar destacado a nivel nacional.

En Chiapas, después de la agricultura, la ganaderfa es la actividad de mayor importancia dentro del sector primario. En 1970 esta rama

4. Véase: Evaluación Plan Chiapas 1983-1986. En otra información de la SARH se indica que de 1984 a 1987 los incendios forestales han destruido más de 280 mil hectáreas. La mayoría de selvas y bosques, (Ambar, octubre de 1987, p.3). 
productiva representó el 29.8 por ciento del PIB sectorial, en 1975 el 32.2, y en 1980 aportó el 27.8 por ciento. A pesar de las variaciones que se observan en la contribución de la ganadería en la generación del PIB sectorial, sobre todo en el periodo 1975-1980, en términos del crecimiento se puede apreciar un ritmo ascendente, ya que mientras en el periodo 1970-1975 la tasa promedio anual fue de 3.1 por ciento, cifra superior al de la agricultura, en el periodo 1975-1980 ascendió al 4.2 por ciento.

Desde mediados de los afios sesenta la ganadería bovina tiende a ser cada vez más importante por el crecimiento de su población, llegando a contar con 2 millones de cabezas en 1970, que significa cerca del 8.0 por ciento de las existencias nacionales; a partir de este afio, y hasta 1980 , la tasa de crecimiento promedio anual fue de 5.7 por ciento, con lo cual se llega a tener una población de $\mathbf{3 . 6}$ millones de cabezas, representando el 10.4 por ciento de las existencias nacionales. Las cifras oficiales indican que en el periodo 1980-1983, la tasa de crecimiento promedio anual es de 3.8 por ciento, por lo que en números absolutos la población ganadera pasa a poco más de 4 millones de cabezas en 1983; con esta cifra, Chiapas se convierte en el segundo estado, después de Veracruz, de mayor importancia en la contribución a la población ganadera nacional con el 10.8 por ciento. Considerando el periodo 1970-1983, la ganadería bovina en Chiapas registra un índice de crecimiento de 202 por ciento, cifra considerablemente superior a la nacional que fue de 149.3 por ciento.

En términos de la producción de came bovina tenemos un crecimiento del 13.9 por ciento en el periodo 1980-1983. Sin embargo, las cifras de producción estarían indicando la persistencia de un modelo de explotación extensivo, en cuanto que las tasas de extracción no superan el 14 por ciento, que significa un promedio inferior al que se obtiene a nivel nacional que es de 17 por ciento.

Hay que reconocer, no obstante, que en los últimos afios se han introducido cambios tecnológicos en las explotaciones ganaderas, tales como pastos mejorados, fertilizantes, inseminación artificial, razas mejoradas, corrales de engorda, etc., sin embargo, no han sido suficientes para elevar la productividad; por el contrario, se observa una continuidad en el crecimiento horizontal de la ganadería, lo cual se refleja claramente en la ampliación de la frontera ganadera que se pone de manifiesto en las pérdidas del recurso forestal y menor crecimiento de la frontera agrícola, particularmente en la región norte y selva lacandona. Tan sólo para tener una idea muy general de las pérdidas del recurso forestal, y de acuerdo con datos de la Delegación Estatal de la SARH, se estima que en los últimos 5 
afios, de 1982 a 1986, se han perdido 90,200 hectáreas del bosque, con un promedio de 18 mil hectáreas por afio 5 .

La superficie ocupada por la ganadería ha venido en ascenso, en tanto que la agricultura y la silvicultura reportan decrementos. Según estimaciones de la SARH, aproximadamente 2.5 millones son de agostaderos, que equivalen a una tercera parte de la superficie del estado. Cifra bastante conservadora pues si consideramos que el índice de agostadero oscila entre una y 1.3 hectáreas por cabeza y el hato ganadero en 4 millones para 1983 , la superficie ocupada por la ganadería no dejaría de ser menos de 3 millones de hectáreas.

De acuerdo con las fuentes oficiales, durante el periodo 1970-1986 la superficie forestal se redujo a un ritmo menor de $1.6 \%$ anual, por lo que durante 16 años han desaparecido poco más de 600 mil hectáreas de bosques tropicales. Cifra poco realista si se toma en cuenta que en ese tiempo se han incrementado las áreas de pastizales y terrenos de pastoreo, la tala para la extracción de madera ha sido constante, el crecimiento demográfico ha ido en ascenso y la construcción de caminos y presas - que aunado a la explotación petrolera, han impactado negativamente en el recurso forestal- se tiene un escenario diferente a los datos oficiales (Informe Final del Estudio del Subsistema de Ciudades, CONPOCIES, 1988).

En base a lo anterior, se puede afirmar que la superficie forestal estimada por la SARH en 3.5 millones de hectáreas no corresponde a la realidad, resultando más congruente la cifra de 2.5 millones de hectáreas reportadas en un estudio reciente (El D La, 28 de febrero de 1983).

Pero el crecimiento de la ganadería bovina se desenvuelve en una serie de contradicciones. Amén de los bajos índices de productividad, la carne se convierte cada vez más en un producto prácticamente inaccesible para la gran mayoría de la población, particularmente la que habita en las áreas rurales; así también, debido a su crecimiento horizontal, genera constantemente conflictos agrarios que con frecuencia se traducen en saldos sangrientos. De acuerdo con datos recientes de la Secretaría de la Reforma Agraria, existen en el estado 71 mil solicitantes de tierra que se ubican preferentemente en las zonas norte, Soconusco y selva lacandona.

La ganadería bovina también crece y se expande a costa de tierras campesinas sobre la vía del rentismo, con lo que se agudiza el problema

5 'El total de hectáreas desmontadas en Chiapas hasta el año de 1978 fue de 2'100,000 hectáreas, de ellas 969,880 se habían dedicado a la agricultura, 741,673 a la ganadería y 388,647 hectáreas eran acahuales". La tala realizada (hasta 1978) en las selvas altas chiapanecas había destruido 525,050 hectáreas, reduciendo sus existencias a 887,245 hectáreas (González, 1983:33). 
de la tenencia de la tierra. En otras palabras, el proceso de ganaderización en Chiapas es y ha sido causa de fuertes conflictos entre campesinos y grandes productores que concentran cada vez más el recurso tierra.

El problema agrario en Chiapas se ha visto agudizado en los últimos años, de tal forma que en 1983 existían más de 100 mil campesinos carentes de tierra, mientras que el $30 \%$ del territorio chiapaneco se encontraba en manos de propietarios privados. En ese mismo ano se denunciaba públicamente la existencia de 2,561 latifundios de explotación ganadera, cuyos dueños se han protegido a través de poderosas asociaciones ganaderas (Secretaría de Salubridad y Asistencia, 1984).

Tan fuerte ha sido el problema agrario en la entidad que los mismos ganaderos se han encargado de denunciar y protestar por la inseguridad en la tenencia de la tierra. En efecto, el 6 de octubre de 1988 más de 1,000 ganaderos de la zona centro, costa y norte del estado efectuaron un plantón en la capital, Tuxtla Gutiérrez, demandando que se detuvieran las invasiones a la pequef́a propiedad. Ahí mismo se dijo que más de doscientos ganaderos de varias partes del estado, pero principalmente de los municipios de Palenque, Playas de Catazajá, La Libertad, Sabanilla y Yajalón tienen problemas de invasión de sus predios.

El problema agrario, que se ha constituido en el punto neurálgico de la entidad, revela también que la mayor parte de la producción ganadera se efectúa en explotaciones privadas, principalmente en grandes propiedades; tan sólo en 1983, 149 ganaderos controlaban el $80 \%$ de la producción pecuaria estatal.

Por otra parte, en un estudio realizado por la Secretaría de Salud sobre la situación nutricional de la población chiapaneca, se sostiene que en 1983 la producción de carne ascendió a 92,764 toneladas, cubriéndose plenamente el consumo aparente estatal estimado en 24,474 toneladas $^{6}$, que en términos relativos representó el $26.3 \%$ de la producción reportada. Sin embargo, la realidad es diferente por cuanto que la gran mayoría de la población tiene una deficiente alimentación en proteínas de origen animal e incluso en calorías.

El problema nutricional se acentúa aún más en las zonas rurales, principalmente entre la población indígena chiapaneca. En efecto, se estima que más del $60 \%$ de la población rural tiene una dieta basada en la ingestión de maíz, frijol y chile; algunos habitantes la complementan eventualmente con frutas y hortalizas. La leche, carne y huevo, práctica-

6 Para 1983, el consumo aparente per cápita anual, se estimó en 10.6 kilogramos, tomando en cuenta una población de 2.3 millones de habitantes. 
mente no se consumen y aquéllos que lo hacen, lo obtienen básicamente de su unidad de producción. Asimismo se afirma que más del $70 \%$ de los preescolares de las áreas rurales presentan algún grado de desnutrición, lo que a su vez repercute en altas tasas de morbilidad y mortalidad (SSA, 1984:24).

La situación se agudiza en la medida en que no se está produciendo para el consumo local, sino que la mayor parte del ganado se canaliza al Distrito Federal y zonas turísticas, así como para el repasto a regiones engordadoras de las Huastecas y Veracruz, principalmente. Así, por ejemplo, en 1977 la Unión Ganadera de Chiapas reportaba una cifra aproximada de 133 mil becerros que salían anualmente para ser engordados en esas regiones. Incluso algunos informantes opinaban que el ganado pasaba en pie de las Huastecas a Estados Unidos (Fernández y Tarrío, 1983:111).

El bajo desarrollo tecnológico, la ausencia de un proceso de industrialización y el deficiente sistema de comercialización hacen que Chiapas cumpla un papel de productor de materias primas. En este sentido, con la movilización de ganado (para repasto y sacrificio), Chiapas está beneficiando a ganaderos e introductores de otros estados del país, transfiriéndose recursos a los sectores engordadores e industriales.

Hay que seffalar, además, que en los últimos años de la presente década se ha registrado un fuerte tráfico ilegal de ganado proveniente de Guatemala, agudizado por la creciente crisis económica y social que vive el vecino país centroamericano, beneficiándose con ello a los ganaderos e introductores de la frontera sur de México.

Las puertas de entrada más importantes del ganado provenientes de Guatemala son Tapachula y Ocosingo. En este último, el contrabando de ganado se realiza por las comunidades fronterizas de Corozal, Chixoy y Benemérito de las Américas y de ahí se canaliza a Palenque. Además, oficialmente se ha estado importando ganado bovino de este vecino país para regularizar el estructural problema de abasto en la ciudad de México y su área metropolitana; así por ejemplo, el valor de las exportaciones de ganado en pie de Guatemala a México en 1981 y 1982 fue de 17,739 y 10,437 miles de quetzales respectivamente ${ }^{7}$. A mediados de 1988, el gobierno mexicano destinó un crédito de 10 mil millones de pesos para la adquisición de carnes y cueros provenientes de Guatemala y Estados Unidos, principalmente.

Otra contradicción fundamental del proceso de ganaderización en Chiapas es que a pesar de la enorme superficie que ocupa genera muy

7 En 1981 un quetzal equivalía a un dólar; en 1988 la relación de cambio fue de 2.7 quetzales por un dólar y en pesos mexicanos de $\$ 850.00$ por quetzal. 
pocos empleos. Si la comparamos con la producción de café, se puede ver que mientras una hectárea de cafe proporciona entre 100 y 150 jornales, un rancho ganadero con 200 cabezas puede ser atendido por 2 vaqueros.

En síntesis, el fenómeno de la ganaderización en Chiapas ha generado fuertes contradicciones: concentración y monopolio de la tierra, destrucción de bosques y selvas, desplazamiento de cultivos, generación de muy pocos empleos, rentismo ilegal de parcelas ejidales, conflictos agrarios, etc. Esta situación tiende a agravarse cada vez más si tomamos en cuenta que la concentración de tierras se encuentra asociada a la apropiación de los recursos crediticios, insumos, maquinaria, etc. La monopolización de los recursos se acompaña inexorablemente de la concentración de los ingresos, con lo que la pobreza de las grandes mayorfas de la población rural está directamente relacionada con la escasez de recursos productivos derivados de un constante proceso de apropiación y pérdida del medio básico: la tierra (Portilla, 1985:66).

\section{CONSIDERACIONES FINALES}

La ganadería bovina mexicana arrastra desde sus origenes una forma extensiva y atrasada de producción. El modelo de acumulación que ha prevalecido en esta actividad se sustenta preferentemente en el uso de grandes extensiones de tierra, teniendo poca significación la inversión de capitales. Sin embargo, actualmente este patrón de crecimiento extensivo ha entrado en crisis como resultado de la conjunción de cuatro factores básicos: 1) agotamiento de la frontera ganadera; 2) reducción de créditos blandos y elevación de las tasas de interés; 3 ) deterioro de los precios de mercado resultante de la fijación de precios oficiales a la carne y leche; 4) contracción de la demanda efectiva de carne y leche, como consecuencia de la reducción en los salarios reales de grandes sectores de la poblacion ${ }^{8}$.

No obstante que, en general, el patrón de crecimiento extensivo ha entrado en crisis, existen expresiones particulares de este fenómeno. En el caso de Sonora ha conducido a un proceso de "reconversión industrial", en donde el sector de punta viene a ser el proceso de engorda en confinamiento, que demanda insumos industriales y cuyo producto final (cortes tipo americano) se destina a un selecto grupo de consumidores. Sin embargo, en Chiapas la ganadería continúa expandiéndose sobre áreas

8 "Entre 1982 y 1987 el consumo de came de res de los mexicanos (bajo) a la mitad, el consumo de leche a una tercera parte, y otro tanto la came de pollo". Héctor Aguilar Camín. "Después del milagro". Ediciones Cal y Arena, México, 1988. Citado en Nexos 1326, abril de 1989, p. 60. 
agricolas y forestales; aunque este crecimiento será por un tiempo limitado y a riesgo de agudizar aún más las contradicciones económicas, sociales y ecologicas. De hecho, en 1986 el gobiemo chiapaneco dispuso la suspensión de créditos a la ganadería extensiva en la región de la selva lacandona. En este sentido "se mantiene la firme decisión de suspender los apoyos a la ganadería extensiva y de generar proyectos productivos alternativos que permitan, paulatinamente, aprovechar potreros ya existentes con cultivos tropicales, de más alta generación de empleo y con el establecimiento de agroindustrias. En las zonas indigenas donde ya existe ganado se apoyarán proyectos de ganadería intensiva para generar ingresos, que permitan sustituir acciones de roza-tumba y quema"

La ganadería del sur de México y en particular la de Chiapas, a pesar de ser relativamente joven, presenta hoy día serias dificultades para su crecimiento. El espectacular ritmo de expansión que registra desde su inicio condujo a una rápida saturación de las tierras, dificultando mantener el mismo ritmo de crecimiento observado hacia los affos ochenta; esto significa que las posibilidades de seguir creciendo bajo el mismo esquema están cerradas en el corto plazo, lo que coloca a la actividad ganadera en una disyuntiva: estancamiento y recesión o crecimiento "intensivo", a través de la capitalización de los ranchos y la utilización de tecnologfas apropiadas para las condiciones de la región.

En lo que se refiere a la frontera norte, los cambios tecnológicos introducidos a partir de la crisis del modelo extensivo han beneficiado preferentemente a los grandes y medianos ganaderos que cuentan con recursos financieros y enormes extensiones de tierra, que al amparo de los certificados de inafectabilidad agraria, han logrado acumular grandes fortunas, situación que les ha permitido la inversión de capitales en otras ramas de la ganadería como es la avicultura y la porcicultura. Algunos empresarios beneficiados con el desarrollo de la industria engordadora ni siquiera son ganaderos en sentido estricto, pues únicamente se dedican a la compra de ganado, obteniendo para ello volúmenes importantes de crédito en condiciones favorables. Se puede decir que el desarrollo de esta actividad ha beneficiado a un reducido grupo de empresarios que se dedican a surtir el mercado nacional y, eventualmente, el mercado exterior. En estas condiciones, un numeroso sector de pequeffos ganaderos y ejidatarios continúan enfrentando los problemas estructurales de la ganadería. Los recursos con que cuentan son cada vez más pobres por el efecto de la

9 Decisiones de la Comisión Intersecretarial para la protección de la Selva Lacandona, y en su segunda reunión del 11 de octubre de 1986, en Palenque, Chispas. La Jornada, 16 de octubre de 1986. 
sobreexplotación, el uso de prácticas inadecuadas y la nula inversión, cuestion que se traduce en un proceso de descapitalización permanente que, de no operarse cambios en este sector, se irá generando cada vez más un estancamiento en la produccion y productividad.

\section{BIBLIOGRAFIA}

AGUILAR Camín, Héctor. 1988. "Después del milagro". En: Nexos, no. 1326, Abril de 1989. Ediciones Cal y Arena, México.

CARRILLO, Mario M. 1988. Crecimiento y desarrollo económico. El caso de Chiapas, 1982-1988. Universidad Autónoma de Chiapas, Tuxtla Gutiérrez, Chiapas, México.

CAMOU, Emesto y Emma Paulina Pérez. 1985. Crisis agricola y expansión ganadera en México. Centro de Investigaciones en Alimentación y Desarrollo, Sonora, México.

CEPAL. 1973. La industria de la carne de ganado bovino. Ed. Fondo de Cultura Económica, México.

CONPO-CIES. 1988. "Informe final del estudio del subsistema de ciudades Tuxtla Gutiérrez-Tapachula". CONPO-CIES, Chiapas, México.

COPARMEX. 1965. "Estudio Integral preliminar sobre la Ganadería en lazona norte de la República Mexicana". Centro de Investigaciones del Desarrollo. 3 tomos, mimeo, México.

CRUZ, Gerardo. 1978. El proceso de institucionalización de la ganaderla bovina de carne en México. IEEc-UNAM, México.

CHAUVET, Michelle. 1987. "Diagnóstico del sistema ganadero bovino: carne y leche en México, alternativas de desarrollo que ofrece la biotecnología". Tesis profesional, UNAM, México.

FEDER, Ernest, et al. 1982. El desarrollo agroindustrial y la ganaderfa en México. SARH-CODAI, no. 8, México.

FERNÁNDEZ, Luis y María Tarrío. 1983. Ganaderla y Estructura Agraria en Chiapas. Universidad Autónoma Metropolitana-Xochimilco, México.

GARCÍA, María del Carmen. 1983. "Participación del Banco Interamericano de Desarrolloen la Problemática Agroalimentaria de América Latina: 1960-1982". CEESTEM, mimeo. México.

GOBIERNO DEL ESTADO DE SONORA. 1980. Plan de Desarrollo Económico y Social 1980-1985. Sonora, México.

GONZÁLEZ, Cuauhtémoc. 1983. Capital extranjero en la Selva de Chiapas 1863-1982. IIEc., México. 
LÓPEZ Rosado, Diego. 1977. Historia de la Agricultura y de la Ganadería. Ed. Herrero, México.

MADRID, Miguel de la. s/f. Cuarto Informe de Gobierno. Estadísticas de la SARH.

PÉREZ Espejo, Rosario. 1987. Agricultura y ganadería. Competencia por el uso de la tierra. Ed. Cultura Popular- IIEC-UNAM, México.

PORTILLA, Belfor, et al. 1985. Alimentación: dependencia o desarrollo nacional. Ed. Nueva Imagen, México.

Secretaría de Salubridad y Asistencia. 1984. "Diagnóstico nutricional del estado". Tuxtla Gutiérrez, Chiapas. 